\title{
WSN based Low Cost and Low Power EPM Design and Field Micro-Climate Analysis using Recent Embedded Controllers
}

\author{
Seema Ajay Agarkar \\ Student of M.E.by Research \\ STM Nagpur Uni. Nagpur, India
}

\author{
Dr.K.D.Kulat \\ Professor and Head (EC) \\ VNIT, Nagpur, India
}

\author{
Dr. R.V.Kshirsagar \\ Professor(EC) and Dean \\ PCEA, Nagpur, India
}

\begin{abstract}
Environment conditions can be maintained by the computerized control systems, where the system can be operated manually or automatically. By 2020 , it will prove to be the utmost necessity everywhere in the world, particularly for agriculture for field micro-climate control. Today, in agriculture, the net house technology is a proven technology for optimum agricultural yield with most minimum natural resources in the countries with far below average rain fall, such as Israel. The production increases manifold irrespective of all adverse environmental conditions. It is necessary to maintain parameters such as temperature, humidity and luminous intensity under control. However, recent technologies including WSN based Environment Parameter Monitoring (shortly called as WSN-EPM) is very costly affair for developing countries including India. Hence, the challenge is to design low cost and low power WSN - EPM.

It is needed to optimize many factors to achieve low power consumption which is prominently supported by many 8-bit embedded products, including recently launched by Microchip such as PIC16F193X family of microcontrollers (MCUs) featuring Microchip`s enhanced Mid-range 8-bit core-the PIC16F1934, PIC16LF1934, PIC16F1936, PIC16LF1936, PIC16F1937 and PIC16LF1937, announced recently in July 2009.
\end{abstract}

Most of the recent microcontrollers provide on chip A to D conversion facility so that applications like WSN are designed with optimum space and power requirements. [3], [4].

The authors have fabricated a very low cost product for environmental parameter monitoring of a net house after exhaustive study of recently launched embedded microcontrollers which need much less power for its operation and hence suitable for remote or field applications. The low cost product is found to be useful in Wireless Sensor Network application of environment parameter monitoring of a net house. Authors strongly believe that the outcome of such comparative studies is needed strongly by many agriculturists and numerous small and medium scale industries owners who are not much inclined to invest, initially, in research and development activities.

\section{General Terms}

Computer Controller Farming, Precision Farming, Low Cost E-Farming, Low Power Controllers
Keywords: Wireless Sensor Network (WSN), aggregation point, Environment Parameter Monitoring (EPM), Microcontroller, PIC, Sensor node, Low Power Device.

\section{INTRODUCTION}

The computer revolution of the early $80 \mathrm{~s}$ created the opportunity to meet the needs for improved control. Consequently, there have been dramatic improvements in control technology. Today, computerized control systems are the standard for modern greenhouses, with continued improvements as the technology advances. Main components of any control system are measurement, controller, data processing, data acquisition and recording. In the environment control system, each parameter must be maintained continuously within a certain range. It needs a complex control system because there are many conditions that depend on the kind of plant and distribution of parameter values at certain times. A number of sensors, switches, actuators (e.g. fans, ventilator \& sprayer) must be installed in the system. Consequently, there are many devices that must be handled in the operation. The use of a computer system will be absolutely necessary to handle control system in order to maintain temperature and humidity with high accuracy.

The wireless sensor networks are a new class of distributed systems that are an integral part of the physical space they inhibit. The creation of large scale sensor networks interconnecting several hundreds to few thousand sensor nodes opens up several technical challenges and immense application possibilities. Wireless sensor networks have recently come in to prominence because they have applications in business asset management to automation in the transportation and health care industries [7].

A wireless sensor network consists of hundreds or thousands of low cost nodes, which could either, have a fixed location or randomly deployed to monitor the environment. The flowing of data ends at special nodes called base stations. As seen in fig.1, base station links the sensor network to another network (like a gateway) to disseminate the data sensed for further processing. Base stations have enhanced capabilities over simple sensor nodes since they must do complex data processing; this justifies the fact that bases stations have workstation/laptop class processors, and of course enough memory, energy, storage and computational power to perform their tasks well. Usually, the communication between base stations is initiated over high bandwidth links [4], [7]. 
The biggest problem of sensor networks is power consumption, which is greatly affected by the communication between nodes. To solve this issue, aggregation points are introduced to the network. This reduces the total number of messages exchanged between nodes and saves some energy. Usually, aggregation points are regular nodes that receive data from neighboring nodes, perform some kind of processing, and then forward the filtered data to the next hop. Aggregation points are clusters. Sensor nodes are organized into

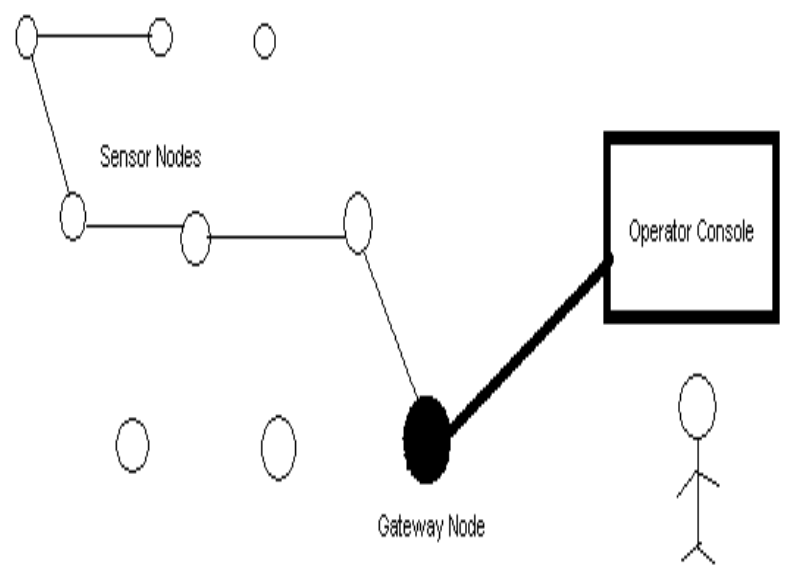

Fig.1. Wireless Sensor Network

clusters, each cluster having a "cluster head" as the leader. The communication within a cluster must travel through the cluster head, which then is forwarded to a neighboring cluster head until it reaches its destination, the base station. Another method for saving energy is setting the nodes to go idle (into sleep mode) if they are not needed and wake up when required [6].

\section{WHY LOW COST LOW POWER WSN- EPM}

The system designed and discussed here is mainly aimed for the developing countries where the economy is agriculturedependent. There are one or several reasons to explain why to use low cost low power WSN-EPM, as given below,

\subsection{Small land holdings}

Most of the farmers in the developing countries, such as, India have very small lands. On an average, farmer in the developing countries has only 2-3 acre of land. This is very negligible as compared to farmers in the developed countries.

\subsection{Poor economic status}

Most of the farmers in the developing countries are poor and have no money to have meal two times a day. These farmers in no way can afford the expensive control systems.

\subsection{Heterogeneity in cropping systems and market imperfections}

Most of the instruments available in the market are manufactured for the soils of the developed countries. It may be possible that these instruments may not function properly in the soil conditions of developing countries. Another reason is the market imperfections, such as, different costs at different places, availability of service centers etc.

\subsection{Illiteracy is the major problem}

Most of the farmers are illiterate and those who are literate don't have much agricultural knowledge. Hence, they are not able to fully utilize their potentials.

\subsection{Uneven natural climatic conditions}

Many a times, due to uneven natural climatic conditions such as droughts, floods, temperature variations and storms, most of the farmers take loan from banks when the farm yield is not good ,thus they suffer from huge bank depts. Sometime these debts lead them to commit suicides even.

\subsection{Monsoon water dependency}

In most of the developing countries, Farmers depend on monsoons for water. If there is ample raining, the farm yield is good else less farm yield is destined. Due to such condition of farmers, it is necessary to bring out the concept of precision agriculture with an emphasis on WSN-EPM.

\section{SYSTEM REQUIREMENTS}

\subsection{Low-cost, low-power, small size networking devices}

The system to be used should be cost effective, small in size and should require less mobility during its functioning. If the system is wireless then it should work on any unlicensed band. It also should require lesser hardware.

\subsection{Long battery life}

Long battery life is needed for the use in developing countries due to frequent power shortage.

\subsection{Robust design}

Developed prototype of WSN-EPM should withstand with variations in the temperature, humidity, rain and wind speed.

\subsection{User friendliness}

Developed prototype of WSN-EPM should have GUI and must be easy to understand and work upon.

In nutshell, socio-economic status of the farmers needs also to be considered while finalising the prototype of WSN-EPM.

\section{AVAILABLE TECHNOLOGIES FOR ENVIRONMENT PARAMETER MONITORING (EPM)}

Plant growth is affected by various factors. The most important factors for the quality and productivity of plant growth are temperature, humidity, light and the level of the carbon dioxide. Continuous monitoring of these environmental variables gives information to the farmer to better understand, how each factor affects growth and how to manage maximal crop productiveness. The optimal greenhouse climate adjustment can enable farmers to improve productivity and to 
achieve remarkable energy savings - especially during the winter in northern countries.

The new tools applicable to the precision farming (PF) are the advances in electronics and computers such as RS, GPS, GIS and WSN, as explained below. Technologies used in precision farming cover three aspects such as data collection, analysis or processing of recorded information and recommendations based on available information, as explained below.

\subsection{Mapping}

The generation of maps for crop and soil properties is the most important and first step in PF. These maps measure spatial variability and provide the basis for controlling spatial variability. Data collection occurs both before and during crop production and is enhanced by collecting precise location coordinates using the GPS. The data collection technologies are grid soil sampling, yield monitoring, RS and crop scouting. During crop production, the data is collected through sensing instruments such as soil probes, electrical conductivity and soil nutrient status. Optical scanners are used to detect soil organic matter and to recognize weeds. Data generated through mapping is recorded and stored in a computer system for future action and generated maps used for acquisition of information and for making strategic decisions to control variability. Mapping can be done by RS, GIS and manually during field operations.

\subsection{Remote sensing (RS)}

It is the acquisition of information about an object from a distance, with precision, without coming into contact with the same. Although the use of RS is a decade old, its relevance to agriculture in spatial variability management is relatively new. RS measures visible and invisible properties of a field or a group of fields and converts point measurements into spatial information, to monitor temporally dynamic plant and soil conditions. The visual observations are recorded through a digital notepad and geo-referenced to GIS database. The satellite images allow mapping of crop, pest and soil properties for monitoring seasonally variable crop production, stress, weed infestation and extent within a field. RS is used for PF in a number of ways for providing input supplies and variability management through decision support system. The point data of soil test results is translated into spatial coverage based on geostatistical interpolation, which gives chemical properties of the soil, nutrient status, organic matter, salinity, moisture content, etc. This information on spatial variability can be used with other georeferences to identify both seasonally stable and variable units, based on which management strategies are developed. Space technology combined with satellite RS and communication provides valuable, accurate and timely information like early warning, occurrence, progressive dangers, damage assessment, quick dissemination of information regarding disaster and decision support to mitigate it.

\subsection{Geographic information system}

Recently, use of GIS in agriculture has increased because of misuse of resources like land, water, etc. GIS is the principal technology used to integrate spatial data coming from various sources in a computer. GIS techniques deal with the management of spatial information of soil properties, cropping systems, pest infestations and weather conditions. This is primarily an intermediate step because it combines the data collected at different times based on sampling regimes, to develop the subsequent decision technologies such as process models, expert systems, etc. The manipulation of spatial information had begun in the 1960s, but has grown rapidly with the development of computer-aided techniques. In the new millennium, GIS-aided techniques are indeed needed for sustainable food production and resource utilization, without further depletion of the environment.

GIS technology helps the farmers and scientists in decision making, as precise information on field is readily available. GIS techniques make weed control, pest control and fertilizer application site-specific, precise and effective; it is also very useful for drought monitoring, yield estimation, pest infestation monitoring and forecasting. GIS coupled with GPS, microcomputers, RS and sensors is used for soil mapping, crop stress, yield mapping, estimation of soil organic matter and available nutrients. In combination, these technologies have brought out rapid changes in data collection, storing, processing, analysis and developing models for input parameters.

\subsection{Wireless sensor networks}

A recent survey of the advances in wireless sensor network applications has reviewed a wide range of applications for these networks and identified agriculture as a potential area of deployment together with a review of the factors influencing the design of sensor networks for this application. WSN is a collection of sensor and actuators nodes linked by a wireless medium to perform distributed sensing and acting tasks. The sensor nodes collect data and communicate over a network environment to a computer system, which is called, a base station. Based on the information collected, the base station takes decisions and then the actuator nodes perform appropriate actions upon the environment. This process allows users to sense and control the environment from anywhere. There are many situations in which the application of the WSN is preferred, for instance, environment monitoring, product quality monitoring, and others where supervision of big areas is necessary. When large number tiny sensor nodes are deployed either randomly or in regular grid, they shall act collectively to perform sensing over a large area or in inaccessible terrains.

Wireless sensor network (WSN) thus forms a useful part of the automation system architecture in modern greenhouses. Wireless communication is used to collect the measurements and to communicate between the centralized control and the actuators located to the different parts of the greenhouse. In advanced WSN solutions, some parts of the control system itself can also be implemented in a distributed manner to the network such that local control loops can be formed. Compared to the cabled systems, the installation of WSN is fast, cheap and easy. Moreover, it is easy to relocate the measurement points when needed by just moving sensor nodes from one location to another within a communication range of the coordinator device. If the greenhouse flora is high and dense, the small and light weight nodes can even be hanged up to the plants' branches. WSN maintenance is also relatively cheap and easy. The only additional costs occur when the sensor nodes run out of batteries and the batteries need to be 
charged or replaced, but the lifespan of the battery can be several years if an efficient power saving algorithm is applied.

The present research paper demonstrates the effective use of WSN concept for precision farming for developing countries with an emphasis on low cost and low power design using recently available embedded controllers.

\section{APPLICATIONS OF WSN}

Sensor networks have been useful in a variety of domains. Some of the important domains are given below.

\subsection{Net house observation}

WSN can be used to monitor net house parameters such as temperature, luminous intensity and most important humidity. Sensor nodes are randomly deployed in and around the net house to know exactly the values of these parameters. The software program in the microcontroller decides when and where the water is to be released so as to control the humidity. Other similar applications of WSN include forest fire detection, air pollution and rainfall observation in agriculture.

\subsection{Area monitoring / military monitoring}

Sensors could monitor vehicular traffic, track the position of the enemy or even safeguard the equipment of the side deploying sensors. In area monitoring, the WSN is deployed over a region where some phenomenon is to be monitored. For example, a large quantity of sensor nodes could be deployed over a battlefield to detect enemy intrusion instead of using landmines. When the sensors detect the event being monitored (heat, pressure, sound, light, electro-magnetic field, etc), the event needs to be reported to one of the base stations, which can take appropriate action (e.g., send a message on the internet or to a satellite). Military uses sensor networks for battlefield surveillance [5], [8].

\subsection{Building monitoring}

Sensors can also be used in large buildings or factories monitoring climate changes. Thermostats and temperature sensor nodes are deployed all over the building area. In addition, sensors could be used to monitor vibration that could damage the structure of a building. Flash microcontroller ATtiny25, ATtiny45 and ATtiny 85 from AVR are useful products as they have the temperature sensor inbuilt in the same chip so that miniature low power WSN can be devised.

\subsection{Healthcare}

Sensors can be used in biomedical applications to improve the quality of the provided care. Sensors are implanted in the human body to monitor medical problems like cancer and help patients maintain their health. Wearable jackets with inbuilt sensors to monitor body parameters are now the part of WSN for quick, mobile and easy patient monitoring.

\subsection{Agriculture}

Using wireless sensor networks within the agricultural industry are increasingly common. Gravity fed water systems can be monitored using pressure transmitters to monitor water tank levels, pumps can be controlled using wireless I/O devices, and water use can be measured and signals are wirelessly transmitted back to a central control center for billing. Irrigation automation enables more efficient water use and reduces waste.

\section{RECENT EMBEDDED PROCESSORS AND EPMN}

New embedded and intelligent processors like 8-bit PIC controllers such as PIC16F193X family of microcontrollers (MCUs) featuring Microchip`s enhanced Mid-range 8-bit corethe PIC16F1934, PIC16LF1934, PIC16F1936, PIC16LF1936, PIC16F1937 and PIC16LF1937, announced recently in July 2009 [4], are widely applicable in wireless sensor networks due to their intelligent features and very low power requirements in the range of a few nano- watts. It is estimated that it will further reduce the price of the sensor network and hence the WSN system will become cost effective thus easily employable in many commercial and non-commercial applications.

\section{USEFUL FEATURES OF THE RECENT MICROCONTROLLERS FOR WSN APPLICATIONS}

The important blocks of microcontroller include mainly reset and brownout detector, analog $\mathrm{I} / \mathrm{O}$ ports and watchdog timer in addition to clock oscillator and real time clock. Clock oscillator executes the program out of the program memory at a certain rate. This rate is determined by the frequency of the clock oscillator. The reset circuit in the controller ensures that at startup all the components and control circuits in the controller start at a predefined initial state and all the required registers are initialized properly. The brownout detector is a

circuit that monitors the power supply voltage. If there is a momentary drop in voltage, the brownout detector resets the processor so that the drop in voltage does not corrupt register and memory contents, which could lead to faulty operation of the controller.

A watchdog timer (WDT) is a special timer with a specific function. It is usually used to prevent software crashes. Once armed, the WDT increments an internal counter at some rate. If the user program does not reset the counter, the counter overflows, which is used to reset the controller. The user software is programmed suitably, therefore, frequently enough, to reset the WDT to give a sort of "I am alive" indication. The assumption is that if the user program does not reset the WDT, it has failed in some way and therefore rather than a system crash or unpredictable system performance, it is better to reset the system. A real timer clock (RTC) is a special timer with the task of maintaining time of day, date, etc. It can be used to time-stamp events.

Most of the recent microcontrollers provide on-chip A to D conversion facility so that applications like WSN are designed with optimum space and power requirements. Such devices are useful for remote and space applications where the batteries are charged using solar energy. Compatible sensors are directly interfaced with analog $\mathrm{I} / \mathrm{O}$ port so as to provide low cost and very low cost solutions and thus the third world countries can take benefit of modern technology for applications like weather forecasting, AWACS and Tsunami warning systems to save hundreds and thousands of lives [3],[4]. 


\section{SELECTION CRITETIA OF SPECIFIC MICROCONTROLLER FOR WSN APPLICATIONS}

Selection of a specific microcontroller is based on the facts, such as, availability of software development aids, market trend and design expertise, power need and speed, instruction set and on chip memory, prototype size and design, I/O and timer availability, possibilities of an application specific integrated circuit (ASIC) based implementation and upward compatibility.

\section{LOW POWER DESIGN CONSIDERATIONS FOR LOW COST ENVIRONMENTAL PARAMETER MONITORING WIRELESS SENSOR NETWORK USING RECENT 8-BIT EMBEDDED CONTROLLERS}

PIC16LC74A exhibits very low power operation which is available with options such as internal resources enabled mode and internal resources disabled mode. This ensures the requirement of the situation in which the WSN is being used. In remote and hostile areas, WSN demands low but continuous power requirement. Hence PIC controllers fulfill such demands without compromising with the quality. It is because of selective conditions of operation, such as sleep mode wherein only required power is drawn from the source to ensure most minimum power consumption and hence power supply unit will have longer life span. Typically, it needs only $0.9 \mu \mathrm{A}$ when WDT and external oscillator of Timer 1 is disabled [4].

PIC16LC74A provides another feature such as it needs very low current when its oscillator works at low frequency. For example, when operated at $32 \mathrm{KHz}$, it draws typical current of $22.5 \mu \mathrm{A}$ and maximum current of $48 \mu \mathrm{A}$. Such feature is very much required in WSN applications where fast response of the sensors is not required. For example, seismic sensors are slow in response and hence higher execution speed is not required. Hence, there is a need of slow clock speed thus low power is drawn. Similarly, on chip A/D converter, when enabled but not used, requires only $90 \mu \mathrm{A}$. Experimental observations given below are emphasizing on the similar benefits due to the use of recent 8-bit controllers.

\section{PARAMETERS TO BE CONTROLLED}

Although the system is a single unit but its use can be seen from various applications, such as,

\subsection{Control of abiotic stress parameters}

Abiotic stress is defined as the negative impact of non-living factors on the living organisms in a specific environment. Abiotic stress is essentially unavoidable. Abiotic stress affects animals, but plants are especially dependent on environmental factors, so it is particularly constraining. Abiotic stress comes in many forms. The most basic stressors include: high winds, extreme temperatures, drought, flood, and other natural disasters, such as tornados and wildfires. The lesser-known stressors generally occur on a smaller scale and so are less noticeable, but they include: poor edaphic conditions like rock content and $\mathrm{pH}$, high radiation, compaction, contamination, and other, highly specific conditions like rapid rehydration during seed germination.

\subsection{Microclimate analysis of crops}

The Microclimate can be defined as the climate close to surfaces, upon and beneath soils, under snow, or in water, on living things (such as trees), or even on individual animals. A microclimate is a local atmospheric zone where the climate differs from the surrounding area. The term is referred to areas as small as a few square feet (for example a garden bed) or as large as many square miles (for example a valley). Microclimates exist, for example, near water bodies which may cool the local atmosphere, or in heavily urban areas where brick, concrete, and asphalt absorb the sun's energy, heat up, and reradiate that heat to the ambient air, the resulting urban heat island is a kind of microclimate. Microclimate mainly depends upon the parameters like temperature, radiation, humidity, $\mathrm{CO}_{2}$ profile and many environmental factors which are directly related to the growth of the plants. Microclimate parameters play an important role in the plant growth and hence the quantity and the quality of the yield.

\subsection{Crop nutrient analysis}

Crop productivity in developing world faces several constraints. One of the major crop productivity constraints in the third world is the unavailability of crop nutrients in appropriate amount and form to crops. Plants require specific amount of certain nutrients in some specific form at appropriate times, for their growth and development. The roles of both macro and micronutrients are crucial in crop nutrition and thus important for achieving higher yields. However, most of our soils are deficient in these nutrients, and must be supplemented through proper crop nutrients management. Crop nutrients could be provided through different nutrients application methods, including fertilizer application to soil, nutrients seed priming and foliar application of nutrients. Each of these application methods has its own advantages and limitations.

\subsection{Crop disease analysis}

Plant disease risk is strongly influenced by environmental conditions. While some animal hosts may provide their pathogens with a consistent range of body temperatures, plant pathogens are generally much more exposed to the elements. Plant disease will tend to respond to climate change, though a number of interactions take place among host, pathogen, potential vectors. In some cases, the actions of land managers may also complicate interpretation of climate change effects. The plant disease management is essential for our continued ability to feed a growing human population. Plant diseases continue to cause serious problems in global food production. Currently more than 800 million people do not have adequate food and at least $10 \%$ of global food production is lost to plant disease. Not only does plant disease affect human food production, it also impacts natural systems.

All these parameters can be controlled directly or indirectly by the use of electronics. In the past few decades, extensive research has been conducted in foreign countries to develop 
systems for controlling these parameters. Many systems are also available in market, but all these systems are very costly. In the present paper, aim of the authors is mostly to develop a cost effective system that could satisfy the need of farmers in the developing countries.

\section{EXPERIMENTAL SET-UP AND HARDWARE REQUIREMENT}

It is expected from the fabricated design of low cost environmental monitoring system of net house for WSN network to give two distinct advantages such as very low power consumption and high speed of operation. It is observed that during simulation process, the ON-OFF control process took average time of 1.11 microseconds per instruction with the maximum clock rate of $12 \mathrm{MHz}$, or $11.1 \mu$ s time for one iteration of 10 instructions. However, to complete one measurement cycle of environmental monitoring application, it has taken around more than 1, 00,000 such iterations. On average, it took around $1,11,000 \mu \mathrm{s}$ time for $1,00,000$ iterations. The simulation process is repeated with PIC microcontroller using MPLAB simulator which took only 1.8 $\mu \mathrm{s}$ for one iteration or $18000 \mu \mathrm{s}$ for $1,00,000$ iterations. The conclusion is that PIC is able to give logic control operation with desired high speed and accuracy as expected in such environmental monitoring applications. Hence, PIC microcontroller is preferred in such processes due to accurate and speedier logic control operation.

In case of WSN, the communication within a cluster must travel through the cluster head, which then is forwarded to a neighboring cluster head until it reaches its destination, the base station. Another method for saving energy is setting the nodes to go idle (into sleep mode) if they are not needed and wake up when required. All PIC parts including PIC16C62A, PIC16C74A ,PIC16F1934, PIC16LF1934, PIC16F1936, PIC16LF1936, PIC16F1937 and PIC16LF1937, announced recently in July 2009 support " sleep mode" facility to save the energy. Hence PIC controllers give two distinct power saving features, one supported due to high clock speed. ASK based transmitter and receiver pair is used to convert wired application into wireless application. Micro C program is downloaded into the microcontroller. Microcontroller can be programmed multiple times and hence tailor-made designs can be made easily available. The cost of the circuit, including sensors, is only 2000 INR per module, as shown in Fig.2A, Fig.2B and Fig.2C. It is easily affordable by the farmers in the developing countries who normally possess small piece of land.

Low Power embedded and intelligent processors (for e.g.8-bit PIC controllers such as PIC16F193X family of microcontrollers or MCUs are having as good as 15 very desirable Features suitable for Remote - Low Power - Cost Effective WSN such as flash program memory up to $14 \mathrm{~KB}$, data RAM up to 512 Bytes , 256 bytes data EEPROM , LCD drive (up to 96 segments), nano-watt technology resulting in sleep currents of $90 \mathrm{nA}$, Watchdog Timer (WDT) current of $500 \mathrm{nA}$ and low-power $32 \mathrm{kHz}$ timer/oscillator current of 600 $\mathrm{nA}$ all at $1.8 \mathrm{~V}$, mTouch sensing solutions peripheral for capacitive touch sensing (16 channels, with support for up to 64 buttons), $32 \mathrm{MHz}$ internal oscillator,16-level hardware stack with overflow/underflow interrupt, up to 14 channels of 10-bit Analog-to-Digital Conversion (ADC) , two rail-to-rail input comparators with 555 Timer operation, 4x8-bit and 1x16-bit timers, with extreme low power Real-Time Clock (RTC) support ,robust and reliable operational monitors, such as Power-On Reset (POR),Brown-out Reset (BOR) and lowpower Watchdog Timer (WDT). Features such as low power watchdog timer, flash program memory, extreme low power RTC , nanowatt technology and 14 channel of 10-bit ADC are very essential features for any low power and remote applications and hence are strongly recommended by the authors for Remote-Low Power-Cost Effective WSN for applications such as Tsunami warning.

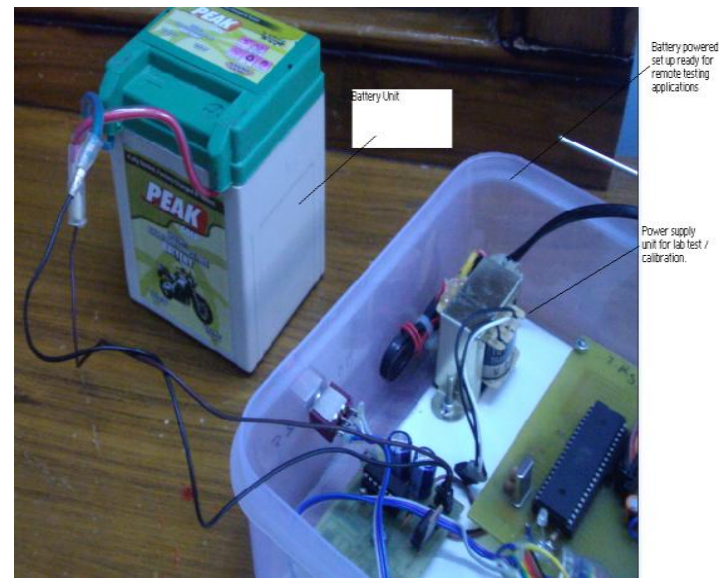

Fig.2A Battery powered unit ready for field testing

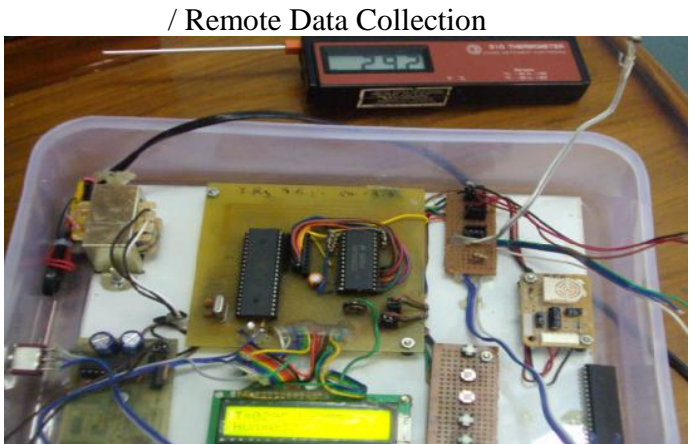

Fig.2B Calibration of the WSN-PME

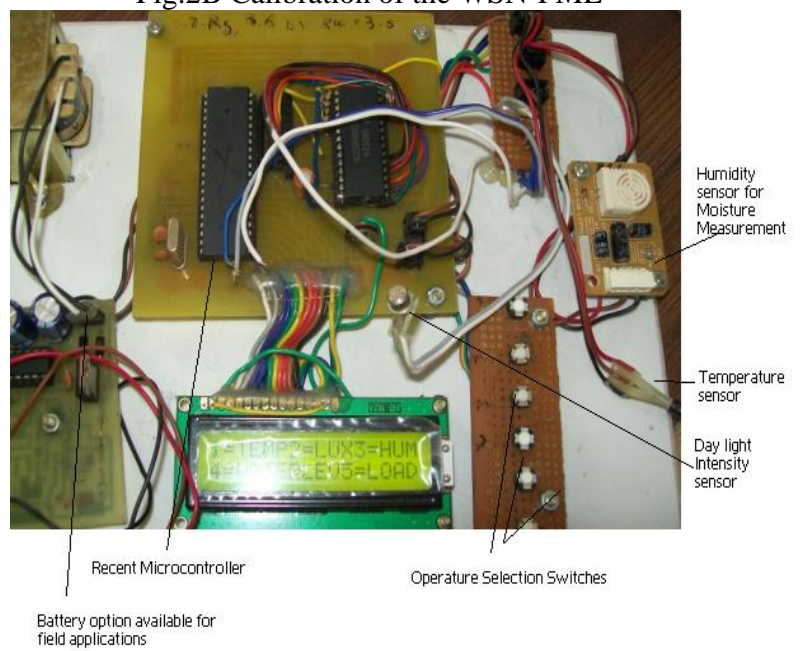

Fig.2C Sensors used in WSN-EPM 


\section{NET HOUSE TECHNOLOGY AND PAYBACK}

The net house technology is a proven technology for optimum agricultural yield with most minimum natural resources in the countries with far below average rain fall, such as Israel. The production increases manifold irrespective of all adverse environmental conditions. Figure 3A and figure 3B show a typical net house in Patur, Amravati Division of the Vidarbha region of Maharashtra (India) for growing of cucumber.

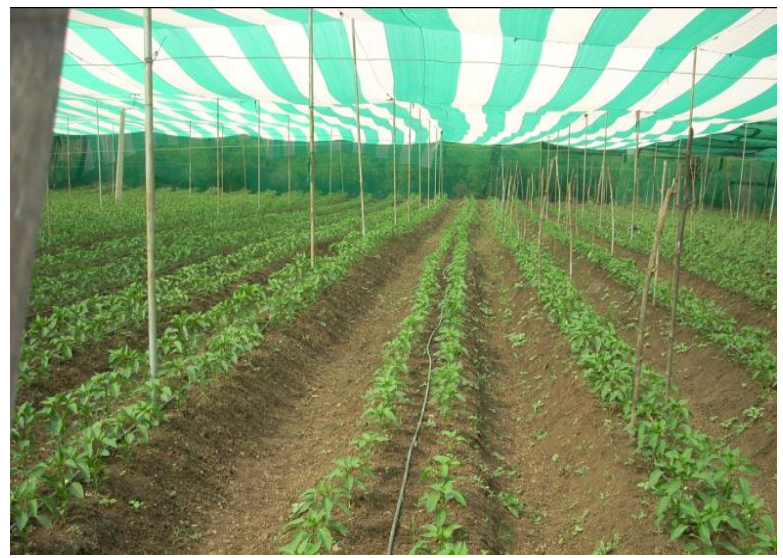

Fig.3A Typical net house in Patur, Amravati Division of the Vidarbha region of Maharashtra for growing of cucumber.

In fact, net houses are meant for farmers with small piece of land and subsidy up to 75000 INR is available from the government to construct a net house for a piece of land measuring up to half of the acre only. The cost of the net house is 125000 INR for half acre of land. However, the parameters such as temperature, luminous intensity (lux) and humidity must be controlled in the ranges specified for optimum yield. Developing countries, such as, India too needs the same technology to be implemented everywhere to increase the agriculture production to satisfy the growing demand of the food grains. Environmental parameter control using WSN can be implemented using recent embedded controllers to provide the measurement and control solution which is very low cost and low power solution. The average construction cost of the net house is INR 2.5 lacs per acre of land. The average cost of one measurement and control module for WSN is around INR 2000 only. Hence for an acre of land, we need 5 to 6 such modules to implement WSN based parameter monitoring of the net house costing around INR 10 to 15 thousand only as compared to Rs. five to six lacs needed for similar equipment supplied by many companies in the developed countries. Hence the fabricated system is easily affordable to the farmers of India and other third world countries. The payback period of the net house cost is 5 to 6 months only.

Benefits are obvious due to this implementation such as controlled irrigation hence saving of water, more frequent irrigation kept surrounding wet and humidity under control, managed water supply to produce high-quality, long and straight cucumber, less total water usage per cucumber tree, yield is almost doubled while maintaining the quality, Average yield went from $250 \mathrm{~kg}$ per day (without WSN -EPM based

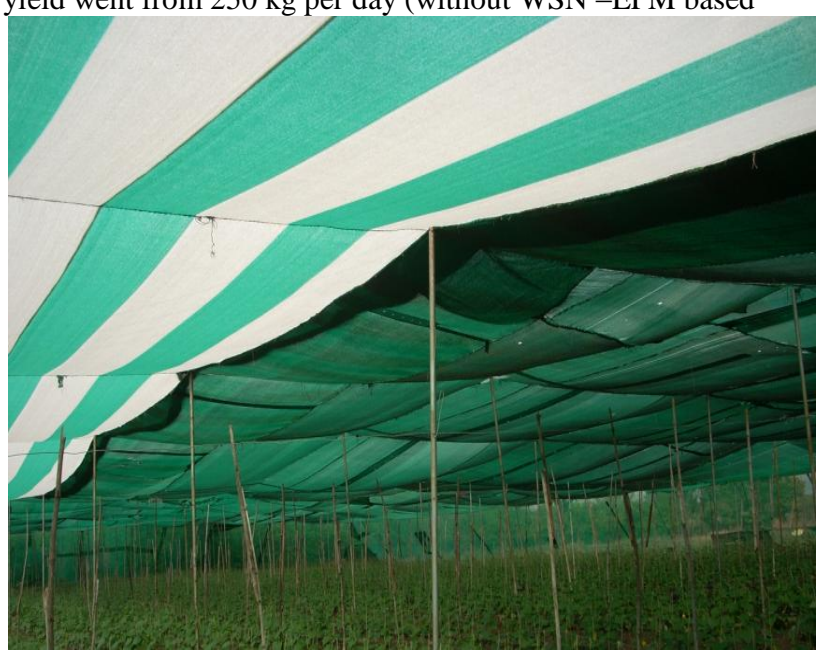

Fig.3B Typical net house in Patur, Amravati Division of the Vidarbha region of Maharashtra (India) for growing of cucumber.

system) to $350 \mathrm{~kg}$ per day (with WSN-EPM based system) for the same size of land. Indirect benefits are including high selling price due to maintained high quality and similarity of the cucumber.

\section{DATA COLLECTION AND RESULTS}

Net house environmental parameter monitoring prototype for WSN is designed using recent microcontroller, ASK based 40 $\mathrm{MHz}$ transmitter and receiver and humidity sensor, temperature sensor light intensity sensor and water level sensors are used to sense the environment. Proper signal conditioning circuits are used to improve the sensitivity of the sensors. Separate power supply unit is used to power the display, signal conditioning and display device. It is seen in Fig. 4A and Fig. 4B that cucumber trees are fully grown to maximize the yield due to proper control of the environmental parameters using WSN-EPM.

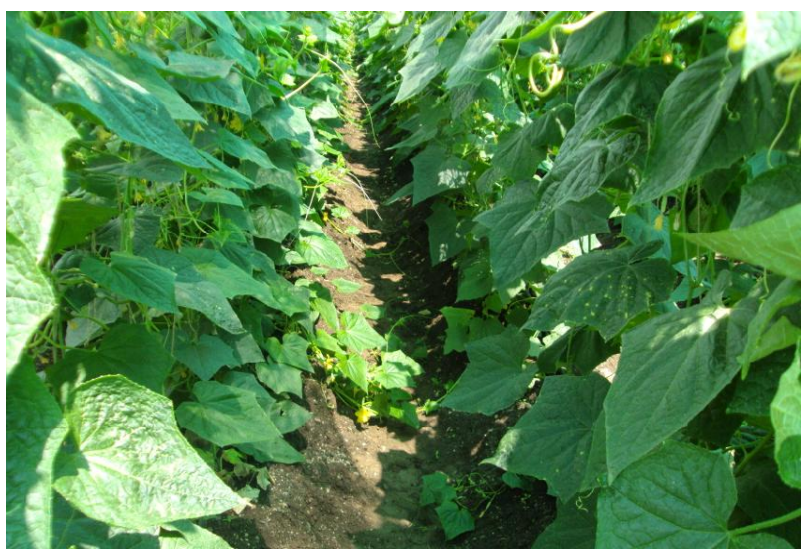

Fig.4A Typical net house in Patur, Amravati Division of the

Vidarbha region of Maharashtra (India) with fully grown cucumber after proper control of the environmental parameters using WSN-EPM. 


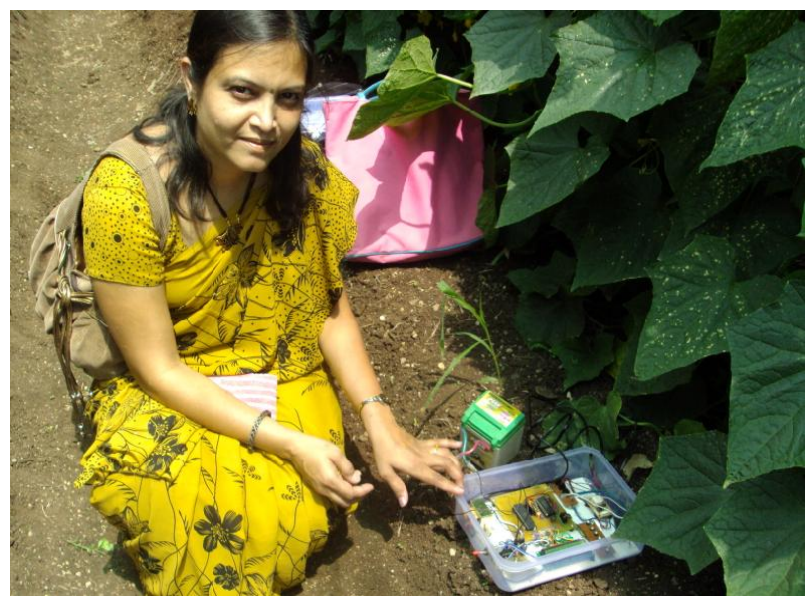

Fig.4B Typical net house in Patur, Amravati Division of the Vidarbha region of Maharashtra (India). Parameter monitoring is being carried out by one of the author using EPM

Data collection is carried out in the field test (green houses shown in Fig. 3A and Fig.3B are used for analysis). Sample data for individual micro-climate parameters is given in Fig.5A through Fig.5L. X- axis shows the time of the day when the particular reading is taken. The sample data produced here is only for one week from 23-08-2009 to 30-08-2009. Time duration is selected to $0830 \mathrm{hrs}(8.30 \mathrm{AM}), 1230 \mathrm{hrs}(12.30$ PM) and 1630 hrs (16.30 PM). In the month of August, September and October, the lux variation is there due to rainy season (cloudy atmosphere). Temperature and humidity is almost normal or near to normal value. A low cost "Microcontroller based Micro- Climate Parameter Monitoring (shortly called as MCPM or EPM) System" designed and developed under the research project work is used to take the readings. Associated control actions are also exercised.

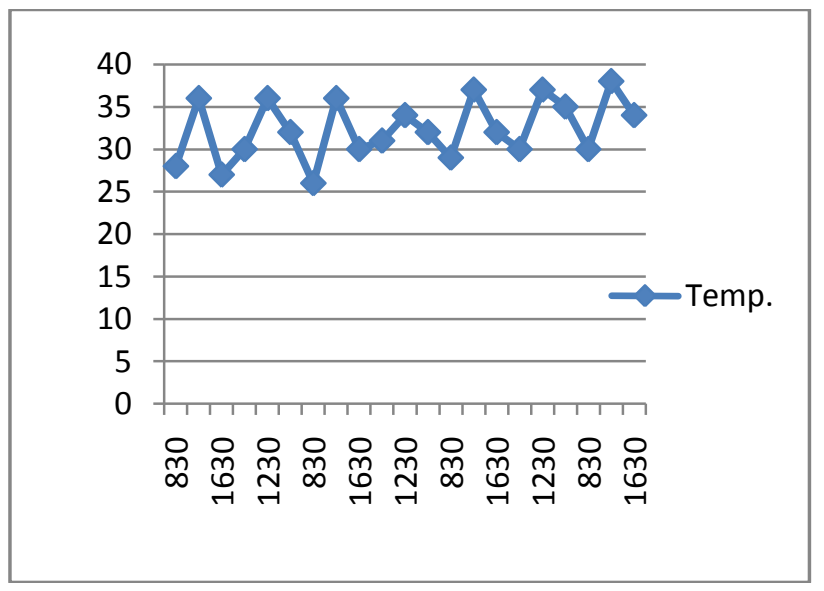

Fig. 5A Variation of temperature in the given week

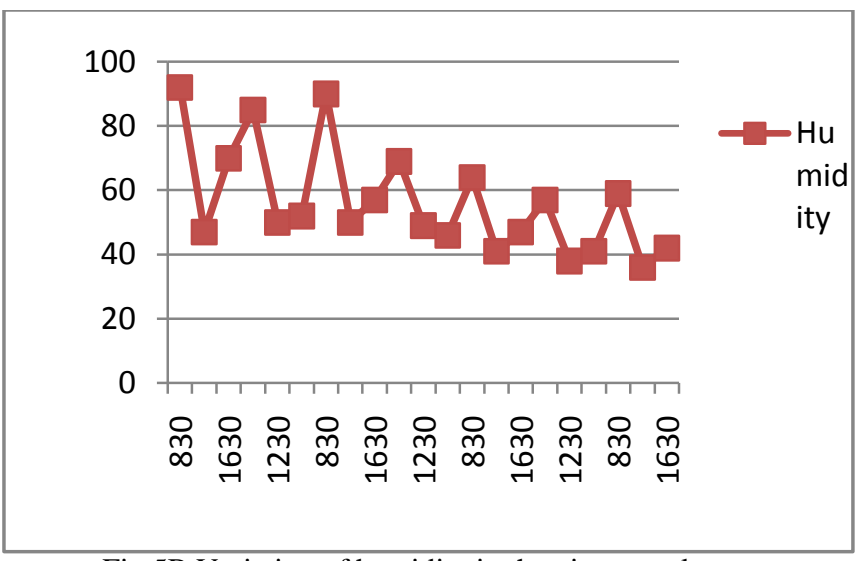

Fig.5B Variation of humidity in the given week

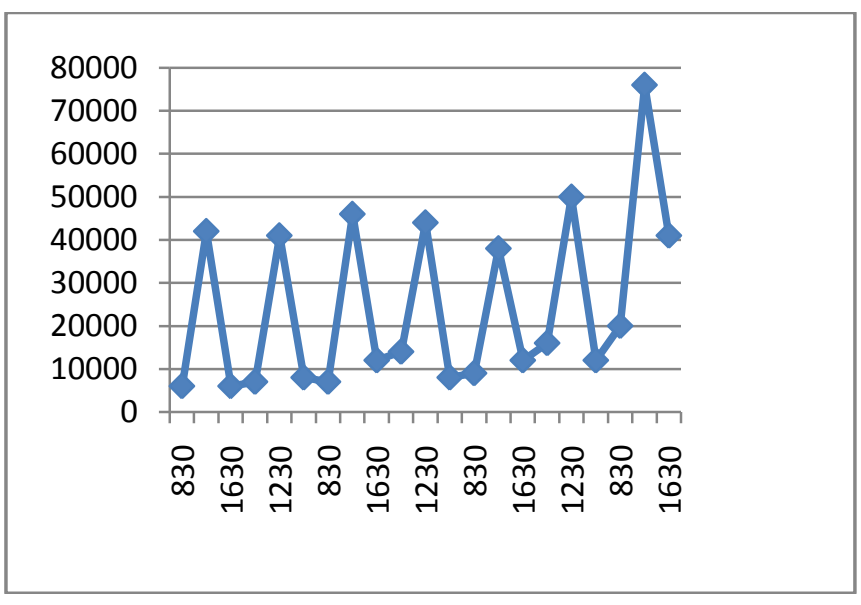

Fig.5C Variation of lux in the given week

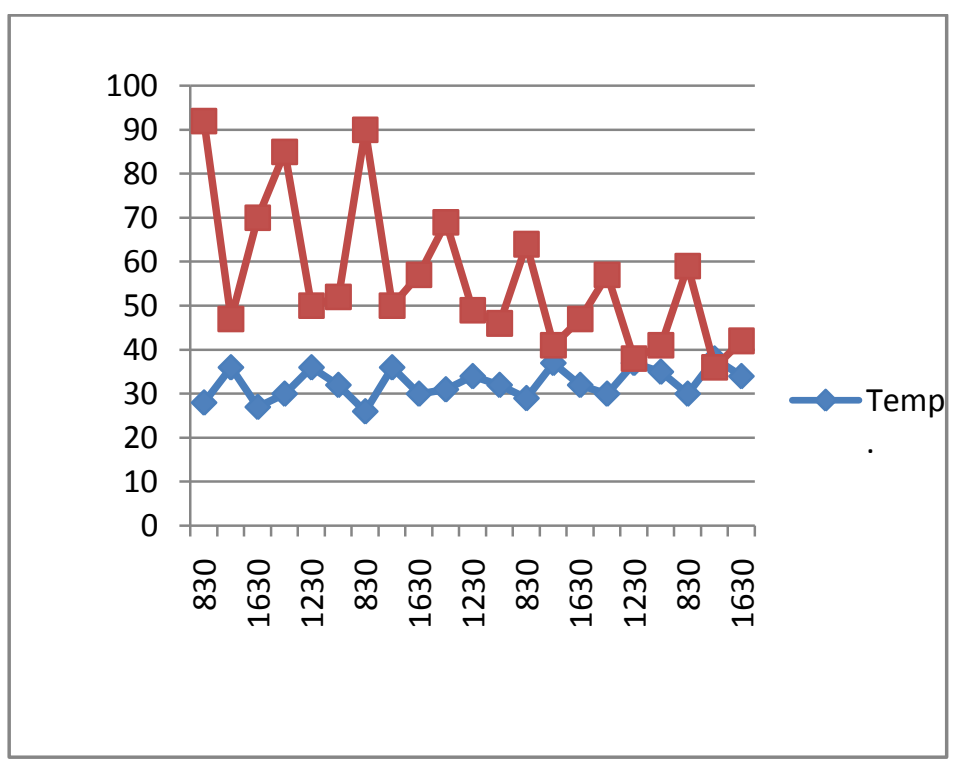

Fig. 5D Comparison of variation of temperature and humidity in the given week 


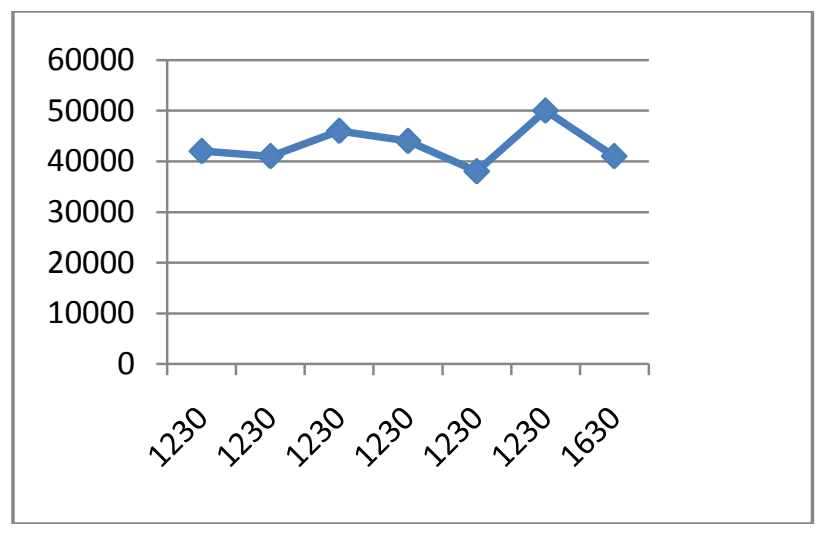

Fig.5E In the given week, the value of Lux is within normal value $(25 \mathrm{k}-55 \mathrm{k}$ lumen) only for 07 times.

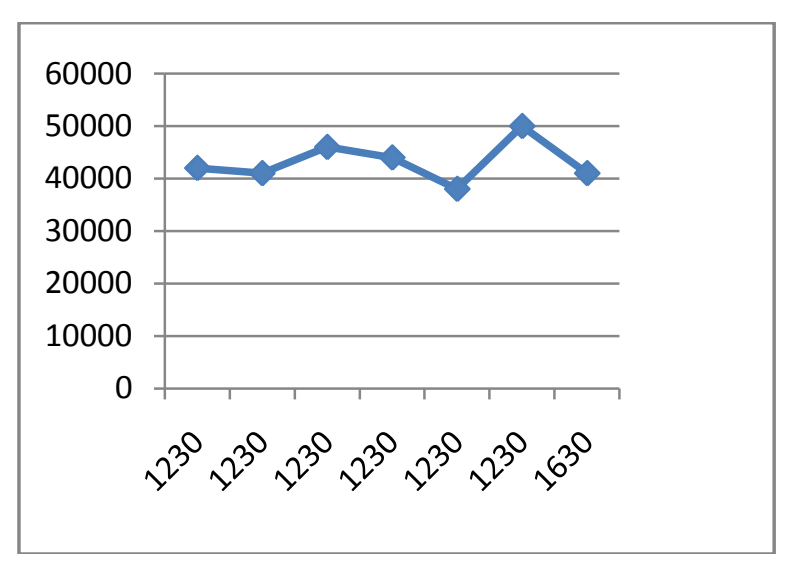

Fig.5F In the given week, the value of Lux is near - to - normal range ( $25 \mathrm{k}-55 \mathrm{k}$ lumen) only for 07 times.

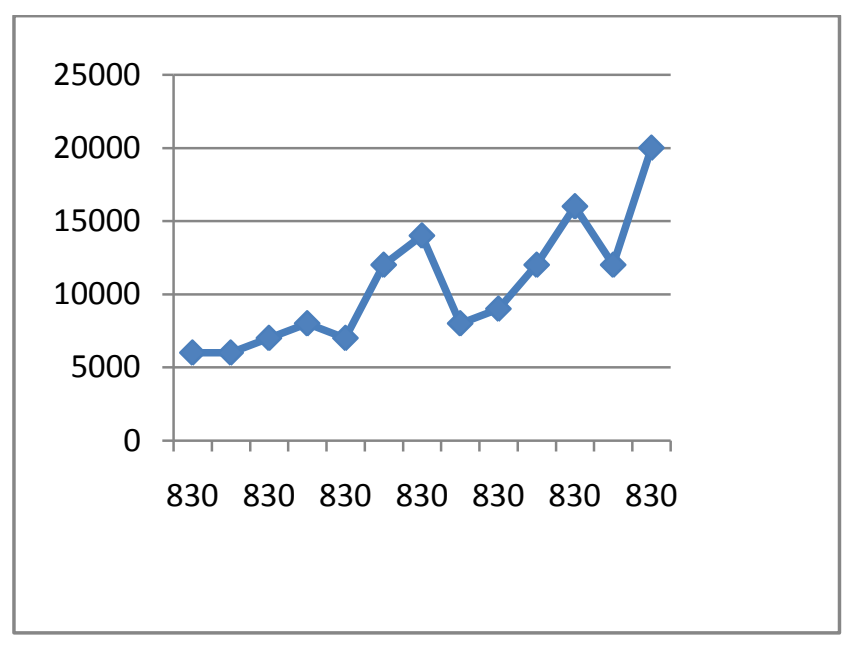

Fig. 5G In the given week, the value of Lux is below normal range $(25 \mathrm{k})$ for many times.

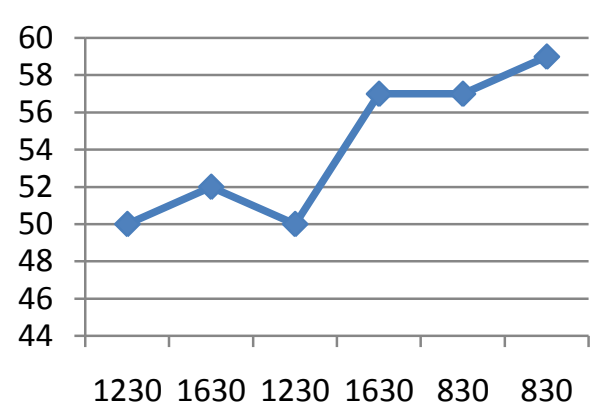

Fig. 5H In the given week, the value of Humidity is within normal range $(50 \%-60 \%)$ for 06 times.

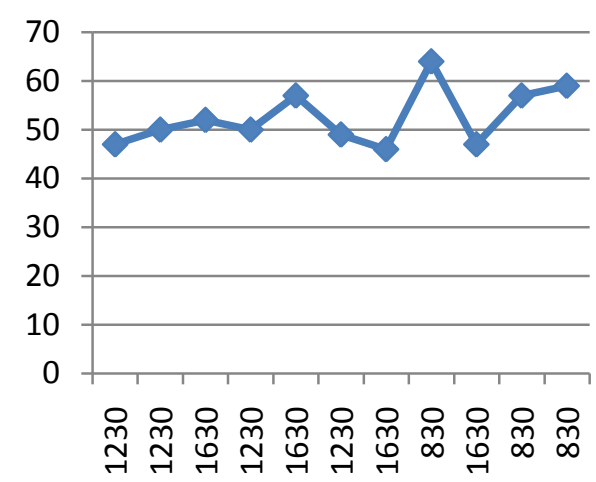

Fig. 5I In the given week, the value of Humidity is near -tonormal range (45\%-65\%) for 11 times.

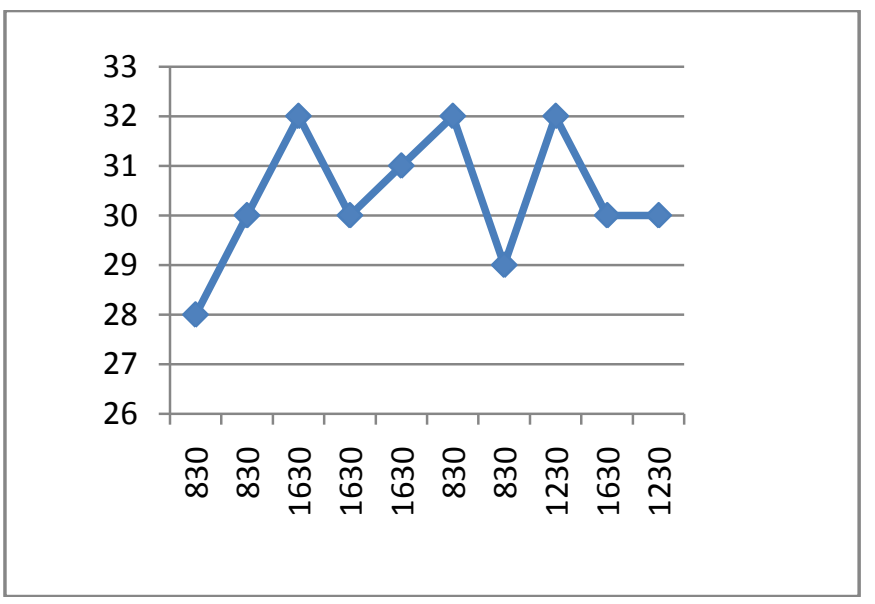

Fig. 5J In the given week, the value of Temperature is within normal $(28 \mathrm{oC}$ to $32 \mathrm{oC})$ values for 10 times 


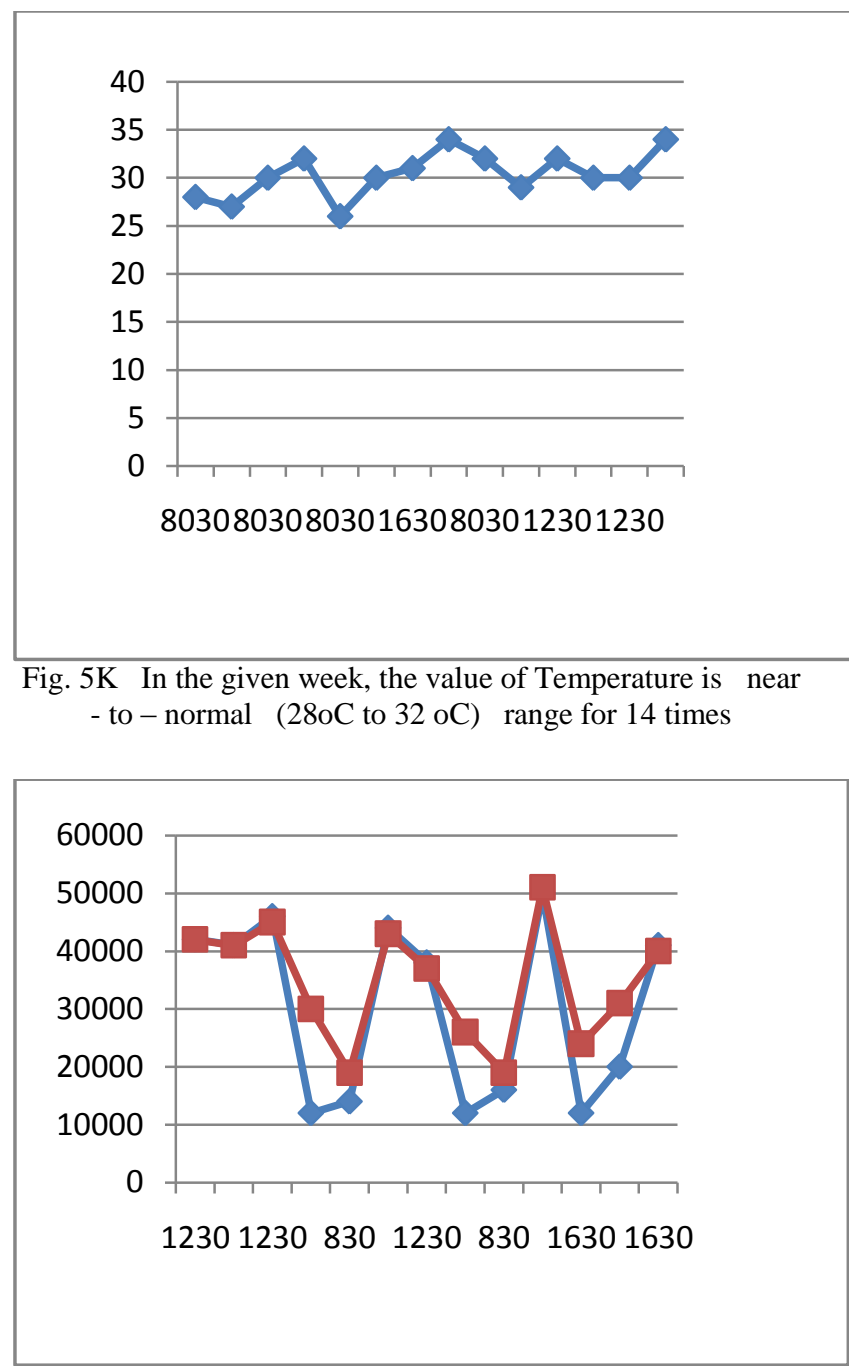

Fig. 5L In the given week, the variation in the lux value is shown. The lux is varied due to corrective action (opening up of the side curtains) when the light intensity was measured with the help of EPM system and found to be low.

\section{RESULT ANALYSIS}

- Cucumber plants covered the area of 1.5 acres.

- Period of observation was 03 months in summer (March, April and May).

- Period of observation was another 03 months in rainy reason and the start of the winter (August, September and October).

- Area of 100 meters by 100 meters was considered as area of observation "a".

- $\quad$ Average Cucumber yield per day from 1.5 acres of area of the excluding " $a$ " is around $250 \mathrm{~kg}$ without micro-climate parameter control.

- Average Cucumber yield (calculated for 1.5 acres if covered under micro-climate parameter control after projecting higher yield from "a") rose from $250 \mathrm{~kg}$ per day to $350 \mathrm{~kg}$ per day.

- Farmers, agriculturists, scientists and entrepreneurs in the meet of Green house owner" club appreciated the system and two green house consultants immediately put the demand for buying the right for the production of such low cost systems.

The prototype developed has shown satisfactory results during field testing. Series of results are taken. Humidity control is precisely obtained only after threshold indication by the device. Farmers were

Satisfied as they can now get an indication using the prototype device

very easily. The agricultural yield has increased as humidity is precisely controlled, even during summer, as compared to random manual control and as expressed by the agricultural experts and the farmers. Lux control is also obtained precisely during rainy season.

\section{CONCLUSIONS}

- PIC16C62A ,PIC16C74A PIC16F1934, PIC16LF1934, PIC16F1936, PIC16LF1936, PIC16F1937 and PIC16LF1937, announced recently in July 2009 support "sleep mode" facility to save the energy. These devices must be preferred by developing countries and third world countries for low power low cost WSN solutions for easy and quick deployment in hostile and remote applications including Tsunami warning systems.

- Advancement of integration between "tiny embedded processors, wireless interfaces, and "micro-sensors" led to emergence of wireless sensor network and its applications manifold for betterment of human life.

- Due to the use of low power PIC microcontrollers, WSN can be used in the space applications to monitor al-Niño effects, cosmic alterations, UFO identification process, green house effects, ozone layer thickness and global warming to lead to concrete implementations at global level for local benefits.

- The prototype developed is designed considering the paying capacity of the farmers from India and other third world countries. Hence it is concluded that the techopreneurs, incubators or STEP managers in these countries can easily bring the prototype into commercial product and bridge the gap between modern technology and the real end users.

- The prototype can later be converted into a low cost farmer's information system so that they needn't seek the help of the expert every now and then.

- Export quality agricultural and horticultural yield is possible due to precise control of humidity. Hence the socio-economic status of the farmers in elevated.

- WSN based system is very much useful for doubling the agricultural yield due to its precision control.

- WSN based system of environmental monitoring prototype is low power and low cost device hence it can be easily implemented in countries like India and other third world countries.

- Maintenance cost is almost zero and hence very much useful for remote applications. 


\section{ACKNOWLEDGEMENTS}

Authors are sincerely thankful to Dr. P.B.Kale, (Additional Director, Vasantrao Naik State Agricultural Extension Management Training Institute, VANAMATI -Institute of Govt. of Maharashtra, situated at Dharmpeth, Nagpur - India) and Mr. A.Y.Talokar, (Assistant Professor, Dept. of Farm Structures, Dr. PKV, Akola - India) for their valuable guidance on the Net Technology. Authors are highly thankful to Hon. Vice-Chancellor Dr. Mayande of Dr. P.K.V.,Akola-India for his motivating words and appreciation regarding low cost feature of the prototype developed during his visit to Patur Net House in the month of March 2010. Authors are thankful to Mr. Kabra (Net House Manufacturer, Murtizapur, Dist. Akola - India) and Mr. Mehta ( Poly House and Net House Manufacturer, Mumbai - India ) for showing keen interest in manufacturing and supplying WSN based Net House Parameter control to the farmers at reasonable cost in near future.

\section{REFERENCES}

[1] F. Akyildiz et al., "A Survey on Sensor Networks," IEEE Communications Magazine, vol. 40, no. 8, 2002.

[2] C. Chong and S.P. Kumar, "Sensor Networks: Evolution, Opportunities, and Challenges," Proc. IEEE, vol. 91, no. 8, 2003.

[3] D. Culler, D. Estrin, and M. Srivastava, "Overview of Sensor Networks," Computer, vol. 37, no. 8, 2004.

[4] Q. Jiang and D. Manivannan, "Routing Protocols for Sensor Networks," Proc. 1st IEEE Consumer Comm. and Networking Conf. (CCNC 04), IEEE Press, 2004.

[5] P. Bonnet, J. Gehrke, and P. Seshadri, "Towards Sensor Database Systems," Proc. 2nd Int'l Conf. Mobile Data Management (MDM 01), 2001.

[6] S.R. Madden, M.J. Franklin, and J.M. Hellerstein, "TinyDB: An Acquisitional Query Processing System for Sensor Networks," ACM Trans. Database Systems, vol. 30, no. 1,2005 .

[7] Chih-Chieh Han et al., "A Dynamic Operating System for Sensor Nodes," Proc. Int'l Conf. Mobile Systems, Applications, and Services (MobiSys 05), ACM Press, 2005.

[8] A. Perrig, J. Stankovic, and D. Wagner, "Security in Wireless Sensor Networks," Comm. ACM, vol. 47, no. 6, 2004.
[9] John B. Peatman, Design with PIC Microcontrollers, eighth reprint, Pearson Education, 2004, p.p. 212 - 224.

[10] NASDAQ: MCHP, "Microchip Technology Announces Net Sales and Net Income for Third Quarter Fiscal Year 2007 and Record Quarterly Cash Dividend," Microchip Financial Press Release, Arizona, p.p.1- 6, Thomas Publishing Company, January 31, 2007.

[11] Databook on current PIC Microcontroller Family Product, 2004. p.p. 12 and Databook on 8- bit AVR Microcontroller with 8-k bytes in-System Programmable Flash, 2006. p.p.07 24.

[12] Dhananjay V. Gadre, Programming and Customizing the AVR Microcontroller Design with PIC Microcontrollers, second reprint, TMH, 2004, p.p. 21 - 58.

[13] NASDAQ : ATML, "Atmel Announces Three New Low Power TinyAVR Microcnotrollers with Internal Temperature Sensor Capabilities," Atmel (R) Corporation, San Jose, California, p.p. 1- 4, Thomas Publishing Company (Press Release), February 21, 2005.

[14] NASDAQ : ATML, "Atmel New AT90SC256144RCFT Secure Microcnotroller targets e-Government Applications", Atmel (R) Corporation, San Jose, California, p.p. 1- 3, Thomas Publishing Company (Press Release), November 07, 2006.

[15] NASDAQ : MCHP, "Microchip's Small and Costeffective Motor Control PIC(r) Microcnotrollers", Microchip Technology Inc, Arizona, p.p. 1- 3, Thomas Publishing Company (Press Release), November 01, 2006.

[16] NASDAQ : ATML, “Atmel's Latest ARM 9 Based Microcnotroller Significantly Increases Internal Data Bandwidth", Atmel Corporation, Rousset, France p.p. 1- 3, Thomas Publishing Company(Press Release), December 11, 2006.

[17] Adam Prengler and David Stone, "8-bit MCUs claim half the automobile", Automotive Design Line (Press Release), p.p. 1- 6, November 01, 2005.

[18] NASDAQ : ATML, "Atmel Offers New AVR Microcontrollers with Integrated LCD Controllers and picoPower Technology", Atmel (R) Corporation, San Jose, California, p.p. 1 - 4, Thomas Publishing Company (Press Release), December 19, 2006. 